\title{
ARTIGO
}

do1 https://doi.org/10.22481/praxisedu.v16i41.6520

\section{ASSOCIAÇÃO ENTRE OS NÍVEIS DE AUTOEFICÁCIA E BURNOUT EM PROFESSORES DE EDUCAÇÃO FÍSICA}

\author{
ASSOCIATION BETWEEN SELF-EFFICACY AND BURNOUT LEVELS IN PHYSICAL \\ EDUCATION TEACHERS
}

\section{ASOCIACIÓN ENTRE NIVELES DE AUTOEFICACIA Y BURNOUT EN PROFESORES DE EDUCACIÓN FÍSICA}

\author{
Erika Cristina de Carvalho Silva Pereira \\ Universidade Federal do Pará - Brasil
}

Maély Ferreira Holanda Ramos

Universidade Federal do Pará - Brasil

Edson Marcos Leal Soares Ramos Universidade Federal do Pará - Brasil

\begin{abstract}
Resumo: Este estudo teve como objetivo verificar a associação entre a autoeficácia e os níveis de Burnout de professores de Educação Física que atuam na Educação Básica. Participaram 63 docentes de Educação Física de escolas públicas e privadas do município de Belém (PA) e região metropolitana. Consiste num levantamento de campo (survey), de abordagem quantitativa e de caráter descritivo e inferencial. Como instrumentos de coleta de dados utilizou para caracterização dos participantes um Questionário sociodemográfico e de trabalho; para aferir os níveis de Burnout o Maslach Burnout Inventory (MBI) e a Escala de autoeficácia de professor de Educação Física, para medir os níveis de autoeficácia. Os dados foram analisados a partir do SPSS (Statistical Package for the Social Sciences), versão 24.0. Para realizar a associação entre as variáveis Burnout e autoeficácia aplicaram-se duas técnicas estatísticas inferenciais: a Análise Fatorial (AF) e a Análise de Correspondência (AC). A partir da AF, os professores foram classificados em três grupos, com alta, média e baixa autoeficácia. Os três fatores explicaram, neste estudo, 73,75\% da variância total das respostas dos sujeitos à Escala de autoeficácia. A AC mostrou que professores de Educação Física com alta exaustão emocional, alta despersonalização e baixa realização pessoal, manifestaram baixa autoeficácia enquanto que docentes com alta autoeficácia estão relacionados a média despersonalização, a média e alta realização pessoal e a média exaustão emocional.
\end{abstract}

Palavras chave: Análise de correspondência. Crenças de autoeficácia. Síndrome de Esgotamento Profissional. 


\begin{abstract}
This study aimed to verify the association between self-efficacy and Burnout levels of Physical Education teachers working in Basic Education. Sixty-three Physical Education teachers from public and private schools in the municipality of Belém (PA) and metropolitan region participated. It consists of a field survey (quantitative approach) with a described and inferential character. As data collection instruments used to characterize the participants a sociodemographic and work Questionnaire; to measure Burnout levels the Maslach Burnout Inventory (MBI) and Physical Education Teacher Self-efficacy Scale, to measure teachers' self-efficacy levels. Data were analyzed using SPSS version 24.0. To make the association between the Burnout variables and self-efficacy, two inferential statistical techniques were applied: the Factor Analysis (FA) and the Correspondence Analysis (CA). From FA, teachers were classified into three groups, with high, medium and low selfefficacy. The three factors explained, in this study, $73.75 \%$ of the total variance of the subjects' responses to the Self-efficacy Scale. The CA showed that Physical Education teachers with high emotional exhaustion, high depersonalization and low personal fulfillment, manifested low selfefficacy while teachers with high self-efficacy are related to average depersonalization, medium and high personal fulfillment and average emotional exhaustion.
\end{abstract}

Keywords: Burnout. Correspondence Analysis. Self-efficacy beliefs.

Resumen: Este estúdio tuvo como objetivo verificar la asociación entre la autoeficacia y los niveles de agotamiento de los maestros de educación física que trabajan em educación básica. Participaron 63 docentes de educación física de escuelas públicas y privadas del municipio de Belém (PA) y la región metropolitana. Consiste en una encuesta de campo, con un enfoque cuantitativo y de carácter descriptivo e inferencial. Como instrumentos de recolección de datos, se utilizo um cuestionario sociodemográfico y de trabajo para caracterizar a los participantes; para medir los niveles de Burnout, el Inventario de Burnout de Maslach (MBI), adaptado para maestros y la escala de autoeficácia del maestro de Educación Física, para medir los niveles de autoeficacia de los maestros. Los datos se analizaroncon SPSS, versión 24.0. Para realizar la asociación entre las variables Burnout y autoeficacia, se aplicaron dos técnicas estatísticas inferenciales: AnálisisFactorial (AF) y Análisis de Correspondencia (AC). Desde la AF, los maestros se clasificaronentres grupos, con alta, media y baja autoeficacia. Los três factores explicaron, en este estudio, el $73.75 \%$ de la varianza total de las respuestas de los sujetos a la Escala de Autoeficacia. El AC mostró que los maestros de Educación Física con alto agotamiento emocional, alta despersonalización y baja realización personal, manifestaron baja autoeficacia, mientras que los maestros con alta autoeficácia están relacionados com la despersonalización promedio, la satisfacción personal promedio y alta y el agotamiento emocional promedio.

Palabras clave: Análisis de correspondencia. Creencias de autoeficacia. Síndrome de Burnout.

\title{
Introdução
}

As pesquisas sobre a Síndrome de Burnout começaram nos Estados Unidos, na década de 1970, e foram realizadas principalmente com grupos de profissionais ligados à área de serviços humanos como médicos, psicólogos, assistentes sociais, enfermeiros, policiais, etc. (BENEVIDES-PEREIRA, 2003). Os pesquisadores pioneiros nas investigações sobre o tema foram o psiquiatra Herbert J. Freudenberger, em 1974, e a psicóloga social Christina Maslach, em 1976 (MASLACH; SCHAUFELI; LEITER, 2001, BENEVIDES-PEREIRA, 2003). 
Inicialmente era um problema que não tinha nome e dificilmente se discutia abertamente sobre o assunto ou se reconhecia tal circunstância, visto que era considerado um tabu dentro dessas profissões (SCHAUFELI; LEITER; MASLACH, 2009; MASLACH, 2017).

Também denominada como Síndrome de Esgotamento Profissional no Brasil, a Síndrome de Burnout tornou-se com o passar dos anos, um problema cada vez mais comum e, de certa maneira, foi disseminada na experiência profissional de trabalhadores em diferentes setores de atuação, assim como em muitos países ao redor do mundo (SCHAUFELI; LEITER; MASLACH, 2009; MASLACH, 2017, PEREIRA; FREITAS, 2019). Para Schaufeli, Leiter e Maslach (2009), as raízes do Burnout parecem estar associadas a fatores de desenvolvimento econômico, culturais e aspectos sociais mais amplos que tiveram início nos EUA, nos anos 1960, e cuja repercussão foi global. Segundo os autores, a síndrome foi resultado de transformações aceleradas nas relações sociais e de uma sociedade industrial para uma economia de serviços.

De acordo com a literatura, cinco concepções teóricas têm conceituado o Burnout, a saber: concepção organizacional; concepção sócio-histórica; concepção clínica; psicologia do trabalho e a concepção psicossocial ou social psicológica (CODO, 1999; CARLOTTO, 2002; SANTOS; NASCIMENTO SOBRINHO, 2011). Entretanto, a perspectiva mais consolidada e utilizada nos estudos é a psicossocial ou sociopsicológica de Christina Maslach (DIEHL; CARLOTTO, 2014). Conforme esta abordagem, o Burnout é considerado uma "síndrome psicológica em resposta a estressores interpessoais crônicos no ambiente de trabalho" e possui três dimensões distintas: 1) exaustão emocional, 2) despersonalização ou cinismo e 3) reduzida realização pessoal no trabalho ou sensação de ineficácia (MASLACH; SCHAUFELI; LEITER, 2001, p. 399, tradução nossa).

A exaustão emocional é resultante de uma carga excessiva de trabalho e está associada ao estresse individual. Caracteriza-se por sentimentos de esgotamento dos recursos físicos e emocionais. Na despersonalização ou cinismo o profissional distancia-se psicologicamente dos destinatários dos serviços. Apresenta uma resposta negativa ou inadequada, desapegada e insensível com relação a diferentes aspectos do trabalho, mas principalmente nas relações interpessoais. A reduzida realização pessoal ou sensação de ineficácia representa a dimensão de autoavaliação do Burnout, onde o trabalhador passa a ter sentimentos de incompetência, de inadequação e de falta de realização, afetando sua produtividade no trabalho (MASLACH; SCHAUFELI; LEITER, 2001; SAVAS; BOZGEYIK; ESER, 2014; MASLACH, 2017; PEREIRA; FREITAS, 2019). O Burnout é 
caracterizado pela presença simultânea de altos índices de exaustão emocional, alta despersonalização e baixa realização pessoal no trabalho.

Quase 50 anos depois dos estudos iniciais sobre a síndrome, o Burnout ainda se constitui como um fenômeno que atinge primariamente as áreas de saúde, serviços e educação, que são os espaços onde se concentram a maioria das investigações (MASLACH; SCHAUFELI; LEITER, 2001; MASLACH, 2017, COUTO, 2018). Especificamente no setor da educação, existe um interesse em compreender o esgotamento vivenciado no trabalho dos professores. Isso porque, estudos mostram que os problemas que os professores encontram nos ambientes escolares, em quase todo o mundo, podem levá-los a experimentar o estresse e ao Burnout (DIEHL; CARLOTTO, 2014; SAVAS; BOZGEYIK; ESER, 2014; COUTO, 2018; PIOVEZAN; DAL RI, 2019). A literatura aponta o ensino como uma das atividades profissionais mais propensas à Síndrome de Esgotamento Profissional ou Burnout (COUTO, 2018; HERNANDEZ, 2018; PEREIRA; FREITAS, 2019).

Segundo Piovezan e Dal Ri (2019), a precarização do trabalho docente, marcada pela flexibilização e intensificação, é um dos principais fatores que tem contribuído para o adoecimento destes profissionais. Segundo as autoras, a flexibilização está associada ao aumento das funções e tarefas dos professores enquanto que a intensificação está relacionada com o aumento da quantidade de trabalho. Para Ferreira (2014), essa situação no contexto do trabalho docente gera prejuízos tanto para a saúde do professor quanto para a vida sociofamiliar.

Além disso, os baixos salários levam muitos professores a procurar preencher ao máximo sua carga horária de trabalho, para aumentar a renda. Uma maior carga horária está diretamente associada a um maior número de turmas e uma maior quantidade de alunos a ser atendida. Os professores não têm tempo para cuidar das demandas pessoais, tampouco para refletir sobre sua prática docente. A discrepância entre as expectativas de realização no trabalho e a realidade das exigências que são impostas aos docentes, bem como a sobrecarga de tarefas, acaba favorecendo o desenvolvimento de problemas emocionais como o estresse, depressão e a Síndrome de Burnout (PIOVEZAN; DAL RI, 2019).

Nessas circunstâncias, encontram-se os professores de Educação Física que ainda precisam lidar com as especificidades do seu componente curricular, com o reduzido apoio recebido dos colegas e da administração da escola; com a escassez de recursos materiais e estruturais para as aulas; com o trabalho em locais inapropriados ou expostos a riscos; com o 
desinteresse dos discentes para participar das aulas, além do baixo status da disciplina na escola (IOCHITE, 2018; PEREIRA; FREITAS, 2019).

Pereira e Freitas (2019) realizaram um estudo com o objetivo verificar os elementos causais e os efeitos da Síndrome de Burnout em docentes de Educação Física a partir de um levantamento bibliográfico. Os autores encontraram um total de 24 artigos, publicados no período de 2004 a 2018, nas bases de dados SCIELO, CAPES e Google Acadêmico, sendo 16 internacionais e 8 nacionais. Constatou-se que o Burnout tem se desenvolvido nos professores de Educação Física a partir de múltiplas variáveis, de origem intrínseca (ex.: idade, sexo, autoeficácia, motivação) e/ou extrínseca (ex.: clima organizacional, satisfação, condições de trabalho). Além disso, verificou-se que apesar das diferenças entre os países de origem das publicações, a síndrome manifesta-se de modo semelhante nestes docentes, prejudicando a vida e o trabalho, e as principais consequências estão associadas à perda de interesse, desistência e abandono da profissão.

No que diz respeito às variáveis intrínsecas ou pessoais, Pereira e Freitas (2019) notaram ausência de estudos correspondentes no Brasil que investigassem, por exemplo, a autoeficácia, a motivação, a paixão pelo trabalho e a associação com a Síndrome de Burnout em professores de Educação Física.

Segundo Maslach (2017), as descobertas sobre Burnout nas últimas décadas sugeriram que fatores ambientais e pessoais são igualmente importantes para a compreensão do desenvolvimento da síndrome nos trabalhadores. Por isso, é fundamental analisar as características pessoais que os protegem dos efeitos do esgotamento, uma vez que não se pode afirmar que todos os professores experimentam Burnout, ainda que, de modo geral, trabalhem sob condições que possam favorecer o desenvolvimento da síndrome (SAVAS; BOZGEYIK; ESER, 2014).

Estudos recentes têm mostrado a autoeficácia como um componente pessoal relevante relacionado à Síndrome de Burnout (HERNANDEZ, 2018; FERREIRA; AZZI, 2011). O conceito de autoeficácia foi originalmente elaborado pelo psicólogo canadense Albert Bandura e refere-se ao julgamento que o indivíduo faz das suas próprias capacidades para lidar com um contexto ou situação específica (BANDURA, 1997; PAJARES; OLAZ, 2008; AZZI; POLYDORO, 2006; SAVAS; BOZGEYIK; ESER, 2014, RAMOS, 2015; FERREIRA et al., 2019). A autoeficácia é considerada um construto central na Teoria Social Cognitiva (TSC) (BANDURA, 1997; IAOCHITE, 2018). A TSC é uma teoria que busca explicar o comportamento humano (AZEVEDO, 1997). 
Bandura (2005) adota na TSC a perspectiva da agência humana, o que significa que o ser humano é um agente, implicando ser capaz de influenciar de maneira intencional o seu próprio funcionamento e as circunstâncias de vida e não apenas ser produto dessas condições. Segundo o autor, as pessoas utilizam os sistemas motor, cerebral e sensorial para realizar suas atividades e alcançar os objetivos que dão satisfação, significado e direcionam suas vidas (BANDURA, 2001).

No caso dos professores, a autoeficácia docente compreende "a crença na própria capacidade de exercer efeito produtivo e relevante na quantidade e qualidade do envolvimento e aprendizagem dos alunos, considerando a diversidade destes" (AZZI; POLYDORO; BZUNECK, 2006, p. 150). Em outras palavras, corresponde à crença que o professor tem nas suas competências e habilidades para ensinar até mesmo os alunos mais difíceis, bem como nas suas capacidades em lidar os desafios no cotidiano educacional.

A autoeficácia funciona como um dos aspectos da personalidade que regulam o afeto, a motivação e a ação, influenciando nas escolhas, na persistência e no esforço empreendido nas atividades realizadas (AZZI; POLYDORO, 2006). Docentes com níveis reduzidos de autoeficácia geralmente evitam os problemas ou tentam resolvê-los mentalmente, favorecendo seu esgotamento emocional. De acordo com Savas, Bozgeyik e Eser (2014) a baixa autoeficácia pode contribuir para o desenvolvimento da ansiedade e estresse, que nos professores estão associados à presença mais elevada da Síndrome de Burnout.

Por outro lado, professores que acreditam ser mais capazes, são mais comprometidos, motivados, se envolvem mais com os alunos, mostram maior satisfação, menos sintomas associados ao desgaste emocional, entendem as adversidades da prática pedagógica como desafios a serem superados e fazem um esforço para resolver os problemas relacionados à profissão (HERNANDEZ, 2018; SAVAS; BOZGEYIK; ESER, 2014; FERNANDEZ, 2015; IAOCHITE, 2018, COUTO, 2018; FERREIRA et al., 2019). A autoeficácia docente pode ser um preditor importante da saúde física e psicológica do professor (HERNANDEZ, 2018; FERREIRA; AZZI, 2011; RAMOS, 2015; COUTO, 2018).

Na Turquia, Yildirim (2015) realizou uma pesquisa com 325 professores de Educação Física, cujo objetivo foi analisar a relação entre Síndrome de Burnout, comprometimento organizacional e o papel mediador da autoeficácia. Para coleta de dados foram aplicadas as escalas de autoeficácia, escala de Burnout, escala de comprometimento organizacional e um questionário demográfico. Os resultados apontaram para uma correlação negativa entre autoeficácia docente e Burnout, ou seja, quanto maiores os níveis de autoeficácia, menores 
foram os índices da síndrome nos professores. Igualmente foi encontrada uma correlação negativa entre Burnout e comprometimento organizacional. A autoeficácia apresentou uma correlação positiva com o comprometimento organizacional, isto é, quanto maior a percepção de autoeficácia dos professores, maior era o compromisso com o trabalho desenvolvido na instituição de ensino.

As pesquisas sobre Burnout, salientam que o esgotamento acarreta custos sérios pessoais, econômicos e organizacionais (MASLACH, 2017). Segundo Gigasari e Hassaskhah (2017), a qualidade da educação é seriamente afetada pelos efeitos do Burnout, uma vez que os docentes se sentem insatisfeitos, não preparados para lidar com o estresse em seu trabalho e estão abandonando a profissão. Além disso, professores com suficientes competências socioemocionais e crenças de autoeficácia elevadas, sofrem menos sintomas relacionados à Síndrome de Burnout (HERNANDEZ, 2018). Considerando o contexto apresentado na literatura, este estudo tem como objetivo verificar a associação entre a autoeficácia e os níveis de Burnout de professores de Educação Física que atuam na Educação Básica.

\section{Metodologia}

\section{Delineamento do estudo}

Este estudo consiste num levantamento de campo (survey), de abordagem quantitativa e de caráter descritivo e inferencial. A pesquisa denominada levantamento de campo caracteriza-se pela realização de coleta de dados junto a pessoas, utilizando diferentes recursos (SILVEIRA; CÓRDOVA, 2009). A abordagem quantitativa consiste em apresentar resultados que podem ser quantificados e utiliza da linguagem matemática para explicar as causas de um fenômeno, assim como as relações entre as variáveis envolvidas (SILVEIRA; CÓRDOVA, 2009). O caráter descritivo está associado ao propósito de descrever os atributos de um objeto de estudo, seja ele fenômeno ou população, além de estabelecer relações entre variáveis analisadas (GIL, 2008). É um estudo inferencial, porque visa obter uma afirmação sobre uma população a partir dos dados de uma amostra (REBULA, 2017).

\section{Participantes}

Participaram da pesquisa 63 professores de Educação Física que lecionam em escolas públicas e particulares do município de Belém e região metropolitana, no Estado do Pará. 
Destaca-se que a amostra foi do tipo não probabilística, por conveniência, e teve como critério de inclusão ser professor (a) de Educação Física, atuando na Educação Básica. A pesquisa tem aprovação do Comitê de Ética da Universidade Federal do Pará (UFPA), sob o Parecer $\mathrm{N}^{\circ}$ 3.026.005. Todos os procedimentos éticos foram realizados conforme a resolução $\mathrm{N}^{\circ} 196$ do Conselho Nacional de Saúde, no que corresponde aos cuidados na pesquisa com seres humanos.

A maioria dos docentes era do sexo feminino, correspondendo a $61,9 \%$ dos participantes, enquanto os homens constituíram 38,09\% do total dos entrevistados. No que concerne ao estado civil, $60,3 \%$ dos professores são solteiros. A faixa etária dos participantes variou de 20 a 61 anos, com idade média de $\pm 36,1(\mathrm{DP}=8,69)$. A maioria dos professores atua exclusivamente em escolas públicas $(69,8 \%)$ e apenas $23,8 \%$ em escolas privadas, enquanto $6,3 \%$ trabalham em escolas públicas e privadas.

\section{Instrumentos para coleta de dados}

Utilizaram-se três instrumentos para a coleta de dados: Questionário de Sociodemográfico de trabalho; Escala de autoeficácia de professor de Educação Física e o Maslach Burnout Inventory - MBI (versão para professores).

O Questionário Sociodemográfico e de trabalho teve como objetivo coletar dados para caracterizar os participantes quanto às informações individuais (sexo, idade, estado civil e formação acadêmica) e de trabalho (tipo de instituição em que leciona, carga horária semanal de trabalho, tempo em que exerce a profissão e renda salarial). Esse instrumento foi construído com base em questionários utilizados em estudos como os de Bernardini (2017), Sinott (2013) e Iaochite (2007).

A Escala de Autoeficácia do professor de Educação Física (EAED) teve por finalidade mensurar os níveis de autoeficácia dos docentes participantes. Esta escala foi adaptada e traduzida por Polydoro et al. (2004), considerando as características do trabalho dos professores de Educação Física (IAOCHITE et al., 2011; VENDITTI JÚNIOR, 2005). De acordo com Iaochite (2007), a adaptação foi feita a partir da Ohio State Teacher Efficacy Scale, das autoras Tschannnem-Moran e Woolfolk Hoy.

A EAED é uma escala do tipo Likert de 6 pontos, constituída de 24 questões ou itens e subdividida em duas subescalas - a da Eficácia da Intencionalidade da ação docente e a da Eficácia do Manejo de classe. O escore da EAED em sua totalidade pode variar de 24 pontos, 
no mínimo e 144 pontos, no máximo. Com média de 84 pontos (IAOCHITE, 2007; RAMOS et al., 2018)

A eficácia na Intencionalidade da ação docente corresponde à crença que o professor tem na sua capacidade de ensinar e de mobilizar os alunos para a realização das atividades (RAMOS et al., 2018). Esta dimensão é formada por 14 itens $(2,4,6,9,10,11,12,14,15$, $17,18,19,20$ e 23), dentre os quais estão "Quanto você pode fazer para ajudar seus alunos (as) a pensar criticamente?”, "Quão bem você pode implementar estratégias alternativas na sua aula?" e "Quanto você pode fazer para ajustar suas atividades ao apropriado nível individual dos seus alunos?".

A eficácia no Manejo de classe é formada por 10 itens (1, 3, 5, 7, 8, 13, 16, 21, 22 e 24) e representa a crença do docente "sobre a sua capacidade de gerenciar os múltiplos aspectos do cotidiano da aula" (RAMOS et al., 2018, p. 55). Nesta dimensão estão questões como "Quanto você pode fazer para que os alunos (as) sigam as regras da aula?", "Quanto você pode fazer para lidar com os alunos (as) mais difíceis?" e "Quanto você pode fazer para controlar comportamentos perturbadores na aula?".

Em estudos anteriores, o $\alpha$ de Cronbach encontrado no processo de validação da Escala de autoeficácia foi de $\alpha=0,937$ na escala geral, $\alpha=0,913$ na Eficácia da Intencionalidade da ação docente e $\alpha=0,863$ na subescala Eficácia no Manejo de classe (VENDITTI JÚNIOR, 2005; IAOCHITE, 2007).

O Maslach Burnout Inventory (MBI - versão para professores) - é uma escala autoaplicável, composta por 22 questões, do tipo Likert de 7 pontos, que indica a frequência com que o indivíduo apresenta determinado sentimento em relação ao trabalho, variando de 0 - nunca a 6 - todos os dias. O MBI é subdividido em três dimensões de avaliação: Exaustão emocional, Despersonalização e Realização pessoal no trabalho (MASLACH; JACKSON, 1981; CARLOTTO; CÂMARA, 2004). Foi traduzido e adaptado para uso com professores brasileiros por Benevides-Pereira et al. (2008) e sua versão original foi elaborada por Maslach e Jackson (1981). Carlotto e Câmara (2004) afirmam que a consistência interna do MBI é satisfatória, visto que apresenta um $\alpha$ de Cronbach de 0,71 até 0,90 e os coeficientes de teste e reteste vão de 0,60 a 0,80 .

No MBI, a exaustão emocional é composta por 9 afirmativas (questões 1, 2, 3, 6, 8, 13, 14, 16 e 20), como por exemplo: "Sinto-me esgotado (a) emocionalmente por meu trabalho" e "Trabalhar com alunos o dia todo me exige um grande esforço". A dimensão despersonalização ou cinismo possui 5 itens (questões 5, 10,11, 15 e 22) e é constituída por 
afirmativas como "Creio que trato alguns alunos com objetos impessoais" e "Tenho me tornado mais insensível com as pessoas desde que exerço este trabalho". A realização pessoal no trabalho conta com 8 itens no questionário (questões 4, 7, 9, 12, 17, 18, 19 e 21) como "Tenho conseguido muitas realizações em minha profissão" e "Sinto que influencio positivamente a vida de outros através do meu trabalho" (CARLOTTO, CÂMARA, 2004; SINOTT, 2013).

\section{Procedimento de Coleta de Dados}

Os instrumentos de coletas de dados e o Termo de Consentimento Livre e Esclarecido foram encaminhados no formato de um formulário Google para os professores de Educação Física de uma turma de especialização por meio de um aplicativo de mensagens. Esses docentes podiam compartilhar o link do formulário com outros profissionais da área por meio do mesmo aplicativo, a fim de que fosse alcançado o maior número de participantes. Além disso, o formulário ficou disponível nas redes sociais dos pesquisadores e do grupo de pesquisa ao qual estão vinculados. Uma vez preenchido, o formulário armazena as repostas automaticamente em uma planilha do Excel, de acesso exclusivo dos pesquisadores.

\section{Procedimento de análise de dados}

\section{Cálculo dos níveis de Burnoute suas dimensões}

Primeiramente foram analisados os níveis das dimensões de Burnout a partir da classificação contida na Tabela 1. Estudos como os realizados por Sinott (2013) e Pires, Monteiro e Alencar (2012) utilizaram os mesmos escores para classificação do Burnout em professores de Educação Física.

Tabela 1 - Classificação dos índices das dimensões de Burnout.

\begin{tabular}{lccc}
\multicolumn{1}{c}{ Dimensões } & \multicolumn{3}{c}{ Pontos de Corte } \\
\cline { 2 - 4 } & Baixo & Médio & Alto \\
\hline Exaustão emocional & $0-15$ & $16-25$ & $26-54$ \\
\hline Despersonalização & $0-02$ & $03-08$ & $09-30$ \\
\hline Realização Pessoal & $0-33$ & $34-42$ & $43-48$ \\
\hline
\end{tabular}

Fonte: Adaptada de Benevides-Pereira (2001); Pires; Monteiro; Alencar (2012); Sinott (2013). 


\section{Verificação das associações entre as variáveis}

Para realizar a associação entre as variáveis Burnout e autoeficácia aplicaram-se duas técnicas estatísticas inferenciais: a Análise Fatorial (AF) e a Análise de Correspondência (AC). Utilizou-se a AF com o objetivo de criar índices de autoeficácia para diferenciar seus níveis entre baixo, médio e alto senso de autoeficácia, aplicando o software SPSS, versão 24.0. A análise de correspondência foi realizada com o auxílio do aplicativo Statistica, versão 6.0. Em todos os testes, fixou-se $\alpha=5 \%(p \leq 00,05)$ para rejeição da hipótese nula.

\section{Resultados e Discussão}

Neste tópico são apresentados os resultados a) do teste de confiabilidade das escalas de autoeficácia e Burnout; b) da análise fatorial para a construção do índice de autoeficácia e c) da análise de correspondência entre os construtos Burnout e autoeficácia.

\section{Confiabilidade das Escalas de Autoeficácia e Burnout}

Confiabilidade de uma escala significa que a mesma é consistente e reflete o constructo que está sendo medido. Para tanto calculou-se o $\alpha$ de Cronbach (CRONBACH, 1951) que é a medida comum para avaliar a confiabilidade de uma escala. Em geral, uma escala é confiável quando o valor $\alpha$ de Cronbach é maior ou igual a 0,70, valores substancialmente menores indicam uma escala não confiável. Nos resultados da análise da confiabilidade das Escalas para avaliar a Autoeficácia e Burnout em Professores de Educação Física os valores $\alpha$ de Cronbach encontrados foram de 0,95 (Autoeficácia) e 0,66 (Burnout).

Kline (1999) mostra que para testes cognitivos, por exemplo, teste de inteligência, um valor comumente aceito é 0,80 , para testes de habilidade um ponto de corte mais adequado é 0,70, quando se tratar de constructos psicológicos, valores abaixo de 0,70 podem ser esperados, em função da diversidade de constructos que estão sendo medidos.

Com relação à confiabilidade da Escala de Burnout, o resultado da presente pesquisa corrobora com os encontrados no estudo desenvolvido por Carlotto e Câmara (2004). As autoras realizaram uma análise com 563 professores da Educação Básica de Porto Alegre, cujo objetivo foi o de verificar a fidedignidade e validade do MBI. Os resultados mostraram que as dimensões da escala (exaustão emocional, despersonalização e realização pessoal no 
trabalho) explicaram $47 \%$ da variância das respostas, alcançando um nível satisfatório de consistência interna, com $\alpha$ de Cronbach elevado para exaustão emocional, de 0,88 , e de 0,82 para realização pessoal. A dimensão despersonalização apresentou um índice moderado, com $\alpha$ de Cronbach $=0,58$. Concluíram assim, que as subescalas do MBI apresentam confiabilidade interna de moderada a alta (CARLOTTO; CÂMARA, 2004).

Um estudo realizado com uma amostra de 163 professores, de escolas estaduais na Turquia, e que também utilizou MBI, encontrou um $\alpha$ de Cronbach de 0,897 na escala geral, $\alpha=0,895$ na subescala exaustão emocional; $\alpha=0,754$ na despersonalização e $\alpha=0,815$ na realização pessoal (SAVAS; BOZGEYIK; ESER, 2014)

Pesquisa realizada por Iaochite (2007), teve entre seus objetivos identificar e analisar a autoeficácia de 263 professores de Educação Física, de escolas púbicas e privadas, em São Paulo. O autor utilizou a escala de autoeficácia docente adaptada por Polydoro et al. (2004) para mensurar os níveis de autoeficácia dos professores. $\mathrm{O} \alpha$ de Cronbach da escala geral foi de 0,937, o que se aproxima do achado neste estudo. As subescalas Eficácia da Intencionalidade da ação docente e Eficácia no manejo de classe, apresentaram alfa de 0,913 e 0,863, respectivamente. Os resultados do estudo indicaram uma elevada crença de autoeficácia dos professores (IAOCHITE, 2007). A mesma escala foi utilizada no estudo de Iaochite et al. (2011), com uma amostra de 220 docentes de Educação Física, e apresentou os mesmos níveis de confiabilidade. Na pesquisa de Ferreira (2014), com 100 professores de Ensino médio, a escala de autoeficácia docente (POLYDORO et al., 2004) teve um $\alpha$ de Cronbach de 0,922.

\section{Resultado da Aplicação da Análise Fatorial para Construção do Índice de Autoeficácia}

A partir da AF obteve-se um considerável número de correlações com valores do nível descritivo inferiores a 0,05 (5\%) para as variáveis (perguntas) utilizadas na construção do Índice de Autoeficácia, indicando que todas as variáveis (perguntas) são adequadas à aplicação da técnica de Análise Fatorial.

$\mathrm{Na}$ Tabela 2, pode-se observar que os valores da estatística KMO para Índice de Autoeficácia $(\mathrm{KMO}=0,877)$ é superior a 0,50 , indicando que há adequação da Análise Fatorial ao conjunto de variáveis (perguntas). Além disso, o nível descritivo do teste de esfericidade de Bartlett $(p=0,000)$, isso acarreta na rejeição da hipótese de a matriz de correlações ser a uma matriz identidade (Tabela 2). Estes resultados respaldam o emprego da 
Análise Fatorial para a extração de fatores e a estimação dos escores fatoriais e posterior construção do Índice de Autoeficácia.

Todos os valores da Medida de Adequação da Amostra (MAA) para as variáveis (perguntas), necessárias a construção do Índice de Autoeficácia, individualmente encontramse em domínio aceitável para a aplicação da técnica de Análise Fatorial, isto é, todos os valores de MAA são superiores a 0,50 (Tabela 2). $O$ fator obtido consegue restituir $73,75 \%$ da informação do conjunto de variáveis (perguntas), como pode ser observado na Tabela 2. Porém, vale lembrar que o critério utilizado para retenção dos fatores não foi o \%Var restituído e sim o critério de Kaiser. Assim, um fator foi retido pelo critério de Kaiser, ou seja, aquele com autovalor superior a 1, para a construção do Índice de Autoeficácia. Todas as variáveis (perguntas) utilizadas na construção do Índice de Autoeficácia têm sua informação restituída de forma satisfatória pelo fator retido, já que apresentam valores de comunalidade igual ou superior a $0,45(45 \%)$ (Tabela 2$)$.

Todas as variáveis (perguntas) utilizadas na construção do Índice de Autoeficácia apresentam no mínimo correlação moderada $(r \geq 0,50)$ com o fator (índice) retido (Tabela 2).

Assim, a partir dos escores fatoriais (Tabela 1), o Índice de Autoeficácia (Y), é dado por

$\mathrm{Y}=0,356 \times \mathrm{P}_{1}+0,124 \times \mathrm{P}_{2}+0,025 \times \mathrm{P}_{3}+0,190 \times \mathrm{P}_{4}+0,233 \times \mathrm{P}_{5}+0,187 \times \mathrm{P}_{6}+0,013 \times$ $\mathrm{P}_{7}+0,070 \times \mathrm{P}_{8}+0,223 \times \mathrm{P}_{9}+0,086 \times \mathrm{P}_{10}+0,098 \times \mathrm{P}_{11}+0,089 \times \mathrm{P}_{12}+0,082 \times \mathrm{P}_{13}+$ $0,080 \times \mathrm{P}_{14}+0,142 \times \mathrm{P}_{15}+0,029 \times \mathrm{P}_{16}+0,041 \times \mathrm{P}_{17}+0,016 \times \mathrm{P}_{18}+0,048 \times \mathrm{P}_{19}-0,048$ $\times \mathrm{P}_{20}+0,151 \times \mathrm{P}_{21}+0,625 \times \mathrm{P}_{22}+0,148 \times \mathrm{P}_{23}+0,137 \times \mathrm{P}_{24}$.

Onde:

P1 - Quanto você pode fazer para lidar com os alunos mais difíceis?

P2 - Quanto você pode fazer para ajudar seus alunos a pensar criticamente?

P3 - Quanto você pode fazer para controlar comportamentos perturbadores na aula?

P4 - Quanto você pode fazer para motivar alunos que demonstram baixo interesse na atividade?

P5 - Até que ponto você pode fazer com que suas expectativas sejam claras em relação ao comportamento do aluno?

P6 - Quanto você pode fazer com que os alunos acreditem que podem realizar bem as atividades?

P7 - Quão bem você pode responder a questões difíceis feitas pelos seus alunos?

P8 - Quão bem você pode estabelecer rotinas para manter as atividades acontecendo de forma tranquila?

P9 - Quanto você pode ajudar seus alunos a dar valor à aprendizagem?

P10 - Quanto você pode avaliar a compreensão dos alunos sobre o que ensinou?

P11 - Até que ponto você pode criar boas questões para seus alunos?

P12 - Quanto você pode fazer para encorajar a criatividade dos alunos?

P13 - Quanto você pode fazer para que os alunos sigam as regras da aula?

P14 - Quanto você pode fazer para melhorar a compreensão de um aluno que está fracassando?

P15 - Quanto você pode fazer para acalmar um aluno que é perturbador ou barulhento? 
P16 - Quão bem você pode estabelecer um sistema de gerenciamento da aula com cada grupo de alunos?

P17 - Quanto você pode fazer para ajustar suas atividades ao apropriado nível individual dos alunos?

P18 - Quanto você pode utilizar uma variedade de estratégias para avaliação?

P19 - Quão bem você pode evitar que alguns estudantes problemas arruínem uma aula inteira?

P20 - Até que ponto você pode propor uma explicação ou exemplo alternativo quando os alunos estão confusos?

P21 - Quão bem você pode responder a um aluno desafiador?

P22 - Quanto você pode auxiliar os pais a ajudar os filhos a irem bem nas atividades escolares?

P23 - Quão bem você pode implementar estratégias alternativas na sua aula?

P24 - Quão bem você pode providenciar desafios apropriados para alunos muito capazes?

No índice obtido os valores positivos dos coeficientes das variáveis (perguntas), indicam que quanto maior for o valor escore obtido para um determinado professor, maior é sua Autoeficácia. Após a obtenção do Índice de Autoeficácia (Y), foi possível calcular os escores fatoriais para cada professor, por exemplo, para o primeiro professor da base de dados, os seguintes escores foram obtidos,
$\mathrm{Y}=0,356 \times 4+0,124 \times 4+0,025 \times 5+0,190 \times 6+0,233 \times 6+0,187 \times 6+0,013 \times 5+0,070$ $\times 5+0,223 \times 6+0,086 \times 2+0,098 \times 1+0,089 \times 6+0,082 \times 6+0,080 \times 6+0,142 \times 3+$ $0,029 \times 5+0,041 \times 6+0,016 \times 3+0,048 \times 2-0,048 \times 6+0,151 \times 3+0,625 \times 1+0,148$ $\times 3+0,137 \times 5=12,11$.

A partir dos escores fatoriais de cada professor foi realizada a padronização dos valores obtidos, para que os mesmos pudessem ser avaliados em uma escala numérica variando de 0 a 1 ou 0 a 100\%. Assim, para o primeiro professor da base de dados, os seguintes escores padronizados foram obtidos, (i) Escore Padronizado do Índice de Autoeficácia (EPIAE), para o primeiro professor da base de dados

$$
\mathrm{EPICAE}_{1}=\left(\frac{F_{i}-F_{\text {min }}}{F_{\text {max }}-F_{\text {min }}}\right) \times 100=\left(\frac{12,11-8,19}{18,84-8,19}\right) \times 100=36,86 \%
$$

Após a obtenção dos escores fatoriais padronizados de cada professor, foi realizada uma classificação dos professores em três grupos distintos. A classificação foi baseada na teoria dos percentis amostrais (BUSSAB; MORETTIN, 2013). Logo, o conjunto de valores dos escores padronizados, foi divido da seguinte forma: Grupo 1 - Professores com escores de 0 a 46,18\% (grupo de professores com os menores escores, isto é, menor Autoeficácia); Grupo 2 - Professores com escores de 46,19\% a 78,45\%; Grupo 3 - Professores com escores de 78,46 a 100\% (grupo de professores com os maiores escores, maior Autoeficácia). 
Tabela 2: Estatísticas Resultantes da Aplicação da Técnica de Análise Fatorial as Variáveis Necessárias à Construção do Índice de Autoeficácia.

\begin{tabular}{|c|c|c|c|c|c|c|c|c|}
\hline \multicolumn{2}{|l|}{ Variável } & KMO & $\begin{array}{c}\text { Esfericidade } \\
\text { Bartlett }\end{array}$ & $\%$ Var. & MAA & Comum. & $\begin{array}{c}\text { Correlação } \\
(r)\end{array}$ & $\begin{array}{c}\text { Escores } \\
\text { Fatoriais }\end{array}$ \\
\hline Quanto você pode fazer para lidar com os alunos mais difíceis & P1 & \multirow{24}{*}{0,877} & \multirow{24}{*}{$\begin{array}{c}\chi^{2}=1074,45 \\
p=0,000\end{array}$} & \multirow{24}{*}{73,75} & $0,903^{\mathrm{a}}$ & 0,803 & 0,691 & 0,356 \\
\hline Quanto você pode fazer para ajudar seus alunos a pensar criticamente & $\mathrm{P} 2$ & & & & $0,788^{\mathrm{a}}$ & 0,863 & 0,551 & 0,124 \\
\hline Quanto você pode fazer para controlar comportamentos perturbadores na aula & $\mathrm{P} 3$ & & & & $0,718^{\mathrm{a}}$ & 0,554 & 0,519 & 0,025 \\
\hline Quanto você pode fazer para motivar alunos que demonstram baixo interesse na atividade & P4 & & & & $0,902^{\mathrm{a}}$ & 0,671 & 0,595 & 0,190 \\
\hline $\begin{array}{l}\text { Até que ponto você pode fazer com que suas expectativas sejam claras em relação ao } \\
\text { comportamento do aluno }\end{array}$ & P5 & & & & $0,681^{\mathrm{a}}$ & 0,731 & 0,707 & 0,233 \\
\hline Quanto você pode fazer com que os alunos acreditem que podem realizar bem as atividades & P6 & & & & $0,860^{\mathrm{a}}$ & 0,678 & 0,656 & 0,187 \\
\hline Quão bem você pode responder a questões difíceis feitas pelos seus alunos & P7 & & & & $0,914^{\mathrm{a}}$ & 0,585 & 0,601 & 0,013 \\
\hline $\begin{array}{l}\text { Quão bem você pode estabelecer rotinas para manter as atividades acontecendo de forma } \\
\text { tranquila }\end{array}$ & P8 & & & & $0,911^{\mathrm{a}}$ & 0,467 & 0,600 & 0,070 \\
\hline Quanto você pode ajudar seus alunos a dar valor à aprendizagem & P9 & & & & $0,795^{\mathrm{a}}$ & 0,762 & 0,637 & 0,223 \\
\hline Quanto você pode avaliar a compreensão dos alunos sobre o que ensinou & $\mathrm{P} 10$ & & & & $0,876^{\mathrm{a}}$ & 0,708 & 0,778 & 0,086 \\
\hline Até que ponto você pode criar boas questões para seus alunos & P11 & & & & $0,818^{\mathrm{a}}$ & 0,770 & 0,735 & 0,098 \\
\hline Quanto você pode fazer para encorajar a criatividade dos alunos & P12 & & & & $0,898^{\mathrm{a}}$ & 0,780 & 0,769 & 0,089 \\
\hline Quanto você pode fazer para que os alunos sigam as regras da aula & $\mathrm{P} 13$ & & & & $0,912^{\mathrm{a}}$ & 0,679 & 0,739 & 0,082 \\
\hline Quanto você pode fazer para melhorar a compreensão de um aluno que está fracassando & P14 & & & & $0,866^{\mathrm{a}}$ & 0,650 & 0,703 & 0,080 \\
\hline Quanto você pode fazer para acalmar um aluno que é perturbador ou barulhento & P15 & & & & $0,900^{\mathrm{a}}$ & 0,806 & 0,819 & 0,142 \\
\hline $\begin{array}{l}\text { Quão bem você pode estabelecer um sistema de gerenciamento da aula com cada grupo de } \\
\text { alunos }\end{array}$ & P16 & & & & $0,921^{\mathrm{a}}$ & 0,677 & 0,722 & 0,029 \\
\hline Quanto você pode fazer para ajustar suas atividades ao apropriado nível individual dos alunos & P17 & & & & $0,906^{\mathrm{a}}$ & 0,767 & 0,786 & 0,041 \\
\hline Quanto você pode utilizar uma variedade de estratégias para avaliação & P18 & & & & $0,909^{\mathrm{a}}$ & 0,735 & 0,764 & 0,016 \\
\hline Quão bem você pode evitar que alguns estudantes problemas arruínem uma aula inteira & P19 & & & & $0,875^{\mathrm{a}}$ & 0,746 & 0,709 & 0,048 \\
\hline $\begin{array}{l}\text { Até que ponto você pode propor uma explicação ou exemplo alternativo quando os alunos estão } \\
\text { confusos }\end{array}$ & P20 & & & & $0,869^{\mathrm{a}}$ & 0,731 & 0,731 & $-0,048$ \\
\hline Quão bem você pode responder a um aluno desafiador & $\mathrm{P} 21$ & & & & $0,918^{\mathrm{a}}$ & 0,744 & 0,776 & 0,151 \\
\hline Quanto você pode auxiliar os pais a ajudar os filhos a irem bem nas atividades escolares & P22 & & & & $0,893^{\mathrm{a}}$ & 0,911 & 0,739 & 0,625 \\
\hline Quão bem você pode implementar estratégias alternativas na sua aula & $\mathrm{P} 23$ & & & & $0,931^{\mathrm{a}}$ & 0,705 & 0,802 & 0,148 \\
\hline Quão bem você pode providenciar desafios apropriados para alunos muito capazes & P24 & & & & $0,899^{\mathrm{a}}$ & 0,771 & 0,806 & 0,137 \\
\hline
\end{tabular}

Fonte: Elaborada pelos autores (2020) 


\section{Resultado da Aplicação da Análise de Correspondência}

Os valores do nível descritivo ( $p$ ) menores que o nível de significância de 0,05 (5\%) e do Critério Beta $(\beta)$ maior ou igual que 30, indicam que tanto as variáveis como suas categorias são dependentes (Tabela 3). Além disso, pode-se observar que a soma dos percentuais de inércia indica que mais que $70 \%$ da informação foi restituída pela AC. Desta forma todos os pressupostos para utilização da técnica de Análise de Correspondência são satisfeitos.

Tabela 3: Estatísticas Resultantes da Aplicação da Técnica de Análise de Correspondência as variáveis: Burnout, Autoeficácia, Despersonalização, Realização Pessoal e Exaustão Emocional.

\begin{tabular}{l|c|c|c|c}
\hline Variáveis & \multicolumn{1}{|c|}{$\chi^{2}$} & $\beta$ & $\%$ Inércia & $P$ \\
\hline Burnout versus Autoeficácia & 126,06 & 87,72 & 100,00 & 0,000 \\
Burnout versus Despersonalização & 142,15 & 27,97 & 100,00 & 0,000 \\
Burnout versus Realização Pessoal & 151,72 & 86,64 & 100,00 & 0,000 \\
Burnout versus Exaustão Emocional & 46,04 & 4,44 & 100,00 & 0,000 \\
\hline Autoeficácia versus Despersonalização & 21,18 & 17,26 & 100,00 & 0,003 \\
Autoeficácia versus Realização Pessoal & 57,57 & 24,57 & 100,00 & 0,000 \\
Autoeficácia versus Exaustão Emocional & 86,83 & 31,88 & 100,00 & 0,000 \\
\hline Despersonalização versus Realização Pessoal & 51,72 & 39,19 & 100,00 & 0,000 \\
Despersonalização versus Exaustão Emocional & 84,45 & 46,50 & 100,00 & 0,000 \\
\hline Realização Pessoal versus Exaustão Emocional & 93,16 & 53,81 & 100,00 & 0,000 \\
\hline
\end{tabular}

Nota: $\chi^{2}$ - Valor do Qui-quadrado; $p$ - Nível Descritivo e $\beta$ - Valor do Critério Beta.

Fonte: Elaborado pela autora (2019)

Na Tabela 4, por sua vez, pode-se observar que professores de Educação Física com Burnout manifestaram baixa autoeficácia. De modo semelhante, estudo desenvolvido por Ferreira (2014), teve como finalidade investigar as relações entre autoeficácia, realização pessoal no contexto da profissão docente e as condições de atuação profissional. Participaram da pesquisa 100 professores do Ensino Médio, de Minas Gerais. Como instrumento para coleta de dados a autora utilizou questionário de caraterização, uma escala de Burnout e um escala de autoeficácia docente. Os resultados apontaram uma correlação negativa entre os níveis de autoeficácia docente com as três dimensões do Burnout, ou seja, quanto mais baixa a autoeficácia, maior foram os índices da síndrome nos participantes. Professores com maior percepção de autoeficácia apresentaram menores escores de Burnout. 
Tabela 4: Probabilidade de associação entre as categorias do Burnout e a Autoeficácia, resultantes da aplicação da técnica estatística multivariada Análise de Correspondência.

\begin{tabular}{l|l|c|c}
\hline \multirow{2}{*}{ Variáveis } & \multirow{2}{*}{ Categorias } & \multicolumn{2}{|c}{ Burnout } \\
\cline { 3 - 4 } & & Sim & Não \\
\hline \multirow{3}{*}{ Autoeficácia } & Baixa & $100,00^{*}$ & $\Phi$ \\
& Média & $T$ & $94,36^{*}$ \\
& Alta & $T$ & $83,73^{*}$ \\
\hline
\end{tabular}

\section{Nota:}

*Probabilidades fortemente significativas, pois $\gamma \times 100 \geq 70 \%$.

$\Phi$ - Não houve relação estatística entre essas categorias

Fonte: Elaborada pela autora (2019)

De acordo com Azzi, Polydoro e Bzuneck (2006) o cotidiano do trabalho dos professores está associado com adversidades, fracassos e frustrações que são próprios “à complexa função de ensinar" (p. 156), visto que muitas vezes compreende o trabalho com um elevado número de alunos, falta de apoio dos colegas, reduzida participação da família, distanciamento da administração escolar, entre outros fatores que dificultam a manutenção de um senso elevado de autoeficácia nos docentes.

Souza et al. (2015, p. 750) afirmam que "para a teoria social cognitiva, reações de estresse são vistas em termos de ineficácia percebida de controle sobre as ameaças e exigências físicas e ambientais e que poderia estar associado a uma baixa vitalidade”. Além disso, segundo Savas, Bozgeyik e Eser (2014), uma autoeficácia reduzida pode contribuir para o desenvolvimento de ansiedade e estresse, e estes podem diminuir a eficiência dos sujeitos.

Na Tabela 5, dos resultados obtidos faz-se alguns destaques, pode-se observar que professores de Educação Física com baixa autoeficácia estão relacionados a alta despersonalização, a baixa realização pessoal e alta exaustão. Corroborando estes resultados, estudo realizado por Bernardini (2017), investigou as possíveis correlações entre a autoeficácia e a Síndrome de Burnout. Os instrumentos de coleta de dados utilizados foram o MBI e a Escala de autoeficácia docente (POLYDORO et al., 2004). Participaram da pesquisa 356 docentes do Ensino Superior. Os resultados mostraram associações significativas entre a autoeficácia e o Burnout, destacando que os professores com menor crença de autoeficácia tinham mais propensão à síndrome.

Professores de Educação Física com alta autoeficácia estão relacionados a média despersonalização, a média e alta realização pessoal e a média exaustão emocional (Tabela 5). Nesse sentido, Carlotto et al. (2015) afirmam que professores com autoeficácia elevada são capazes de atenuar os efeitos da sobrecarga laboral originadas das diferentes atividades que envolvem a atividade docente. Além disso, os autores destacam que os professores que creem 
na sua capacidade em lidar com as variadas demandas da profissão, tendem a manifestar menor esgotamento psicológico.

Tabela 5: Probabilidade de associação entre as categorias da Autoeficácia e as categorias da: Despersonalização, Realização Pessoal e Exaustão Emocional, resultantes da aplicação da técnica estatística multivariada Análise de Correspondência.

\begin{tabular}{l|l|c|c|c}
\hline \multirow{2}{*}{ Variáveis } & \multirow{2}{*}{ Categorias } & \multicolumn{3}{|c}{ Autoeficácia } \\
\cline { 3 - 5 } Despersonalização & Baixa & Baixa & Média & Alta \\
\hline \multirow{3}{*}{ Realização Pessoal } & Média & $\Phi$ & $74,41^{*}$ & $\Phi$ \\
& Alta & $97,94^{*}$ & $\Phi$ & $98,41^{*}$ \\
& Baixa & $100,00^{*}$ & $\Phi$ & $\Phi$ \\
\hline \multirow{3}{*}{ Exaustão } & Média & $\Phi$ & $64,83^{*}$ & $95,49^{*}$ \\
& Alta & $\Phi$ & $55,75^{*}$ & $81,86^{*}$ \\
\hline & Baixa & $\Phi$ & $100,00^{*}$ & $\Phi$ \\
& Média & $\Phi$ & $\Phi$ & $100,00^{*}$ \\
& Alta & $98,32^{*}$ & $\Phi$ & $\Phi$ \\
\hline
\end{tabular}

\section{Nota:}

*Probabilidades fortemente significativas, pois $\gamma \times 100 \geq 70 \%$.

T - Não houve relação estatística entre essas categorias

Fonte: Elaborado pela autora (2019)

Segundo Arata (2008), a autoeficácia é considerada como um fator de redução e alívio do estresse, possibilitando que o indivíduo se sinta à altura dos desafios que enfrenta, de modo que a ansiedade, o medo e a incerteza que enfrente em seu contexto de atuação, estejam em níveis que não interferem em seu desempenho. $\mathrm{O}$ autor ratifica ainda que a autoeficácia não é apenas um fator protetor da Síndrome de Burnout, mas também está associada com a percepção de um maior êxito no cumprimento da tarefa pedagógica.

A alta autoeficácia associada aos níveis médios das dimensões de Burnout, nos resultados deste estudo (Tabela 5) pode dar indícios de que os professores, apesar das circunstâncias estressoras e desafiadoras no ambiente de trabalho, estão tentando utilizar estratégias para realização de suas tarefas laborais e a fim de alcançar seus objetivos educacionais. Ferreira e Azzi (2011) afirmam que sujeitos com uma alta percepção de autoeficácia decidem resolver os problemas enfrentados utilizando mecanismos que melhoram seu trabalho, em contrapartida, pessoas com um senso de autoeficácia ocupacional reduzido, apresentam estratégias inadequadas para aliviar a tensão.

Ferreira (2014) reitera que professores com uma autoeficácia maior identificam os problemas e são mais perseverantes na busca de soluções. Observa-se nesse aspecto, que tal constatação não corrobora a ideia de que o professor deve tomar sobre si a responsabilidade de resolver todos os problemas da educação. Porém, o docente pode investigar e reconhecer 
as possibilidades de minimizar os fatores estressores em seu contexto imediato, uma vez que, segundo Bandura (1997) a autoeficácia é mediadora entre a identificação dos obstáculos e as ações necessárias para a resolução.

De acordo com Azzi (2014), é preciso lembrar que acreditar nas próprias capacidades não é tudo. Para que ações efetivas aconteçam, outras dimensões são requeridas, como o desenvolvimento de habilidades e a motivação para a ação. Uma vez que, na TSC, o comportamento humano é multideterminado (AZZI, 2014) por fatores pessoais, ambientais e comportamentais, é preciso pensar nas crenças de autoeficácia apenas como uma parte dos aspectos que influenciam o desenvolvimento da síndrome de Burnout nos professores de Educação Física.

Sendo assim, é fundamental que questões como as condições de trabalho dos professores sejam pensadas e modificadas. Considerando as especificidades da Educação Física como, por exemplo, o baixo status da disciplina no contexto escolar; a falta de interesse dos alunos na realização das aulas; o reduzido apoio dos colegas e administração escolar; a insuficiência de recursos materiais e a ausência ou precariedade de estrutura física para prática das aulas (IOCHITE, 2018; COSTA FILHO; IAOCHITE, 2018), faz-se necessário que políticas públicas de prevenção e combate sejam elaboradas e implementadas.

Maslach (2017) afirma que o contexto local de trabalho é especialmente significativo e não deve ser ignorado em nenhuma tentativa de encontrar soluções para o Burnout. Além disso, a autora destaca a relevância de se investigar as relações sociais no ambiente de trabalho, uma vez que estudos recentes identificaram que locais de trabalho socialmente tóxicos, caracterizados por conflitos não resolvidos, medo, incivilidade, falta de apoio mútuo e confiança. Pesquisas sugerem ainda, intervenções para melhorar o clima organizacional, a fim de que haja menos absenteísmo e desgaste emocional (MASLACH, 2017; PEREIRA; FREITAS, 2019).

\section{Considerações finais}

Os resultados deste estudo corroboram a literatura recente a respeito da relação entre Síndrome de Burnout e autoeficácia, uma vez que os professores investigados que apresentaram baixos índices de autoeficácia, foram os mesmos que tiveram níveis elevados de exaustão emocional e despersonalização, e reduzida realização pessoal ou sensação de ineficácia, caracterizando a presença da síndrome. Por outro lado, a análise dos dados mostrou 
ainda que os docentes com elevado senso de autoeficácia, apresentaram índices médios nas três dimensões do Burnout.

Tais resultados podem colaborar para a reflexão sobre as características do trabalho do professor de Educação Física, visando contribuir para a reformulação de políticas educacionais que proporcionem melhores condições para o exercício da profissão deste docente, uma vez que têm manifestado a síndrome, podendo repercutir negativamente não apenas sua própria saúde, como também todo o processo de ensino-aprendizagem e consequentemente na qualidade da educação. Além do mais, pode auxiliar na elaboração de programas que favoreçam o aumento das crenças de autoeficácia docente, uma vez que esta pode ser um fator protetor contra o desenvolvimento da Síndrome de Burnout.

Sugere-se que outras pesquisas sejam realizadas com uma amostra maior de professores, e em diferentes regiões do país, a fim de que os resultados traduzam uma maior representatividade da realidade dos professores de Educação Física. Do mesmo modo, recomenda-se que estudos de abordagem qualitativa e com outros instrumentos sejam efetuados para uma melhor compreensão das variáveis que contribuem tanto para a progressão do Burnout quanto para os baixos índices de autoeficácia nos docentes.

\section{REFERÊNCIAS}

ARATA, Manuel Fernández. Burnout, autoeficácia y estrés em maestros peruanos: tres estúdios fácticos. Ciência \&Trabajo, ano 10, n. 30, p. 120-125, 2008.

AZEVEDO, Mário. A Teoria Cognitiva Social de Albert Bandura. Universidade de Lisboa, Faculdade de Ciências. Lisboa, 1997.

AZZI, Roberta Gurgel. Introdução à Teoria Social Cognitiva. São Paulo: Casa do Psicólogo, 2014. 130 p. (Série Teoria Social Cognitiva em contexto educativo, v.1).

AZZI, Roberta Gurgel; POLYDORO, Soely Aparecida Jorge.; BZUNECK, José Aloyseo Considerações sobre a auto-eficácia docente. In: AZZI, R. G.; POLYDORO, S. A. J. (org.) Auto-eficácia em diferentes contextos. Campinas, Editora Alínea, 2006.

AZZI, Roberta Gurgel; POLYDORO, Soely Aparecida Jorge (org.) Auto-eficácia em diferentes contextos. Campinas, Editora Alínea, 2006.

BANDURA, Albert. Self-efficacy: the exercise of control. New York: Freeman, 1997 BANDURA, Albert. Social cognitive theory: anagentic perspective. Annual Reviews Psychologist, v. 52, p. 1-26, 2001.

BANDURA, Albert. The Evolution of social cognitive theory. In: SMITH, K. G.; Hitt, M.A. Great minds in management. Oxforf University Press, 2005, p. 9-35. 
BENEVIDES-PEREIRA, Ana Maria Teresa. O estado da arte do Burnout no Brasil. Revista Eletrônica Interação Psy, v. 1, n. 1, p. 4-11, 2003.

BENEVIDES-PEREIRA, Ana Maria Teresa; YAEGASHI, Solange Franci Raimundo; ALVES, Irai Cristina Boccato; LARA, Silvana de. O trabalho docente e o Burnout: um estudo em professores paranaenses. Anais... Curitiba: PUC, 2008, p. 4870-4884.

BERNARDINI, Priscile. Estudo correlacional sobre autoeficácia e Burnout no trabalho docente no Ensino Superior. 103f. Dissertação (Mestrado em Educação). Universidade do Oeste Paulista, Unoeste, Presidente Prudente, 2017. Orientadora: Prof. ${ }^{a}$ Dr. ${ }^{a}$ Camélia Santina Murgo.

BUSSAB, Wilton de Oliveira; MORETTIN, Pedro Alberto. Estatística Básica. 8.ed., São Paulo: Editora Saraiva, 2013.

CARLOTTO, Mary Sandra. A Síndrome de Burnout e o trabalho Docente. Psicologia em Estudo, v.7, n. 1, p. 21-29. 2002.

CARLOTTO, Mary Sandra; DIAS, Sofia Raquel da Silva; BATISTA, Jaqueline Brito Vidal; DIHEL, Liciane. O papel mediador da autoeficácia na relação entre a sobrecarga de trabalho e as dimensões de Burnout em professores. Psico-USF, Bragança Paulista, v. 20, n. 1, p. 13-23, 2015.

CARLOTTO, Mary Sandra; CÂMARA, Sheila Gonçalves. Análise fatorial do Maslach Burnout Inventory (MBI) em uma amostra de professores de instituições particulares. Psicologia em Estudo, v. 9, n. 3. P. 499-505, set./dez. 2004.

CODO, Wanderley. Educação: carinho e trabalho. 4. ed. Petrópolis: Vozes, 1999

COSTA FILHO, Roraima Alves da; IAOCHITE, Roberto Tadeu. A percepção da capacidade de ensinar: um estudo com professores de Educação Física em início de carreira. In: IAOCHITE, Roberto Tadeu (Org). Teoria Social Cognitiva e Educação Física: diálogos com a prática. São Paulo, CREF/SP, 2018.

COUTO, Andréa Lobato. Adoecimento de docentes na Educação Básica: uma revisão sistemática da literatura. 127f. Dissertação (Mestrado em Educação). Universidade Federal do Pará, UFPA, Belém, 2018. Orientadora: Prof. ${ }^{a}$ Dr. ${ }^{a}$ Maély Ferreira Holanda Ramos.

CRONBACH, Lee Joseph. Coefficient Alpha and the Internal Structure of tests. Psychometrika, v. 16, p. 297-334, 1951.

DÍAZ, Francisca Rius; LÓPEZ, Francisco Javier Barón. Bioestatística. 1. ed. São Paulo: Thomson Learning, 2007.

DIEHL, Liciane; CARLOTTO, Mary Sandra. Conhecimento de professores sobre a Síndrome de Burnout: processo, fatores de risco e consequências. Psicologia em Estudo, Maringá, v. 19, n. 4 p. $741-752$, out./dez. 2014 
FERNANDEZ, Ana Patrícia de Oliveira. Crenças de eficácia de professores no contexto de ensino. 140f. Tese (Doutorado em Teoria e Pesquisa do Comportamento). Universidade Federal do Pará, Belém, 2015. Orientador: Prof. Dr. Fernando Augusto Ramos Pontes

FERREIRA, Luiza Cristina. Mauad. Crenças de autoeficácia docente, satisfação com o trabalho e adoecimento. Revista Psicologia: Ensino e formação, v. 5, n. 2, p. 19-37,2014.

FERREIRA, Enizete Andrade; PEREIRA, Erika C. C. Silva; SILVA, Emmanuelle Pantoja; RAMOS; Maely F. Holanda. Autoeficácia em professores alunos do PARFOR-UFPA. TSC em Foco, Bragança Paulista, Ano 1, n. 4, p. 14-28, maio 2019. Disponível em: https://www.teoriasocialcognitiva.net.br/tsc-em-foco/n4/. Acesso em: 01 out. 2019.

FERREIRA, Luiza Cristina Mauad, AZZI, Roberta Gurgel. Docência, Burnout e considerações da teoria da auto-eficácia. Psicologia: ensino \& formação, v. 1, n. 2, p.23-34, 2011

GIGASARI, Niloufar Saber; HASSASKHAH, Jaleh. The effect of social comparison tendencies on EFL teachers' experience of Burnout and instructional self-efficacy. Congent Psychology, n. 4, p. 1-21, 2017. Disponível em:

https://www.researchgate.net/publication/317032708_The_effect_of_social_comparison_tend encies_on_EFL_teachers\%27_experience_of_Burnout_and_instructional_self-efficacy.

Acesso em: 10 out. 2018.

GIL, Antonio Carlos. Métodos e técnicas de pesquisa social. 6. ed. São Paulo: Atlas, 2008.

HERNÁNDEZ, Edgar Torres. Competencias socioemocionales y creencias de autoeficácia como predictores del Burnout em docentes mexicanos. Revista de Estudios y Experiencias em Educación, v. 17, n. 35, p. 15-27, 2018.

IAOCHITE, Roberto Tadeu. (Org). Teoria Social Cognitiva e Educação Física: diálogos com a prática. São Paulo, CREF/SP, 2018.

IAOCHITE, Roberto Tadeu. Auto-eficácia de docentes de Educação Física. 157f. Tese (Doutorado em Educação). Universidade Estadual de Campinas, Unicamp, Campinas, 2007. Orientadora: Prof. ${ }^{a}$ Dr. ${ }^{a}$ Roberta Gurgel Azzi.

IAOCHITE, Roberto Tadeu; AZZI, Roberta Gurgel, POLYDORO, Soely A. Jorge; WINTERSTEIN, Pedro José. Autoeficácia docente, Satisfação e Disposição para continuar na docência por Professores de Educação Física. Rev. Bras. Ciênc. Esporte, Florianópolis, v. 33, n. 4, p. 825-839, out./dez. 2011.

KLINE, Paul. The Handbook of Psychological Testing. London: Routledge, 2.ed., 1986.

MASLACH, Christina. Finding solutions to the problem of Burnout. Consulting Psychology Journal: Practice and Research, v. 69, n. 2, p. 143-152, 2017.

MASLACH, Christina; JACKSON, Susan. E. The measurement of experienced Burnout. Journal of Occupational Behavior, New Jersey, v. 2, p. 99-113, 1981 
MASLACH, Christina; SCHAUFELI, Wilmar B.; LEITER, Michael. P. Job Burnout. Annual Review of Psychology, Palo Alto, v. 52, p. 397-422, 2001.

PAJARES, Frank; OLAZ, Fabián. Teoria Social cognitiva e auto-eficácia: uma visão geral. In: BANDURA, A.; AZZI, R. G.; POLYDORO, S. (Org.). Teoria social cognitiva: conceitos básicos. Porto Alegre: Artmed, 2008. p. 97-114.

PEREIRA, Erika C. C. Silva; FREITAS, Rogério Gonçalves. Panorama global sobre Síndrome de Burnout em professores de Educação Física. Trabalho \& Educação, v. 28, n. 2, p. 97-111, maio-ago. 2019.

PIOVEZAN, Patrícia Regina, DAL RI, Neusa Maria. Flexibilização e Intensificação do Trabalho Docente no Brasil e em Portugal. Educ. Real. Porto Alegre, v. 44, n. 2, 2019.

PIRES, Daniel Alvarez; MONTEIRO, Paulo A. Pimentel; ALENCAR, Diego Rodrigues. Síndrome de Burnout em professores de Educação Física da região nordeste do Pará. Pensar a Prática, Goiânia, v. 15, n. 4, p. 821-1113, out./dez. 2012.

POLYDORO, Soely; WINTERSTEIN, Pedro José; AZZI, Roberta Gurgel; DO CARMO, A. P.; VENDITTI JR, Rubens. Escala de auto-eficácia do professor de Educação Física. In: MACHADO, C. et al. (Org.) Avaliação Psicológica: formas e contextos vol. X. Psisquilíbrios Edições - Braga, 2004, p. 330-337.

RAMOS, Valmor; KUHN, Filipy; BACKES, Ana Flávia; BARROS, Thaís E. BRASIL, Vinícius Z.; SOUZA, Jeferson R.; COSTA, Matheus L.; SOARES, Gabriela F. Percepção de autoeficácia docente: um estudo sobre as experiências de universitários de educação física. $\mathbf{R}$. bras. Ci. E Mov, v. 26, n. 3, p. 53-62, 2018

RAMOS, Maély Ferreira Holanda. Modelo Social Cognitivo de Satisfação no trabalho: percepções sobre a docência. 239f. Tese (Doutorado Teoria e Pesquisa do Comportamento) Universidade Federal do Pará, UFPA, Belém, 2015. Orientador: Prof. Dr. Fernando Augusto Ramos Pontes.

REBULA, Uanderson. Estatística Aplicada. (Série Estatística Industrial). 2017. E-book. Disponível em: https://issuu.com/uandersonrebula/docs/prof._uanderson__estat_stica_aplic_368c45126199d1 Acesso em: 7 nov. 2019.

SANTOS, Alaíde Almeida dos; NASCIMENTO SOBRINHO, Carlito Lopes. Revisão Sistemática da Prevalência da Síndrome de Burnout em professores do Ensino Fundamental e Médio. Revista Baiana de Saúde pública, v. 35, n. 2, p. 299-319abr./jun. 2011.

SAVAS, Ahmet C.; BOZGEYIK, Yumis; ESER, Ismail. A study on the relationship between teacher self efficacy and Burnout. European Journal of Educational Research, v. 3, n. 4, p. 159-166, 2014.

SCHAUFELI, Wilmar B.; LEITER, Michael P.; MASLACH, Christina. Burnout: 35 years of research and practice. Career Development International. V, 14, n. 3, p. 204-220, 2009. 
SILVEIRA, Denise Tolfo; CÓRDOVA, Fernanda Peixoto. A pesquisa científica. In: GERHARDT, T. E.; SILVEIRA, D. T. (Org.). Métodos de Pesquisa. Porto Alegre: Editora da UFRGS, 2009, p. 31-42.

SINOTT, Edilene Cunha. Síndrome de Burnout: um estudo com professores de Educação Física das escolas municipais de Pelotas. 134f. Dissertação (Mestrado em Educação Física). Universidade Federal de Pelotas, UFPel, Pelotas, 2013. Orientadora: Prof. ${ }^{a}$ Dr. ${ }^{a}$ Mariângela da Rosa Afonso.

SOUZA, Luciane Albuquerque Sá; TORRES, Ana Raquel R.; BARBOSA, Genário A.; LIMA, Tiago J. Souza de; SOUZA, Luana E. Cunha. Bem-Estar Subjetivo e Burnout em Cadetes Militares: O Papel Mediador da Autoeficácia. Psicologia Reflexão e Crítica, v. 28, n. 4, p. 744-775, 2015

VENDITTI JÚNIOR, Rubens. Análise de auto-eficácia docente de professors de Educação Física. 148f. Dissertação (Mestrado em Educação Física). Universidade Estadual de Campinas, Unicamp, Campinas, 2005.Orientador: Prof. Dr. Pedro José Winterstein.

YILDIRIM, Írfan. The Correlation between Organizational Commitment and Occupational Burnout among the Physical Education Teachers: the Mediating Role of Self-Efficacy.

International Journal of Progressive Education, v. 11, n. 3, p. 119-130, out. 2015.

\section{SOBRE OS AUTORES:}

\section{Erika Cristina de Carvalho Silva Pereira}

Mestre e Doutoranda em Educação pela Universidade Federal do Pará (UFPA), Belém - PA. Membro do Núcleo de Estudos aplicados ao Comportamento (NEAC). E-mail: erika7carvalho@gmail.com

iD http://orcid.org/0000-0003-0701-9815

\section{Maély Ferreira Holanda Ramos}

Doutora em Teoria e Pesquisa do Comportamento pela Universidade Federal do Pará (UFPA), Belém -PA. Professora permanente no Programa de Pós-graduação em Educação (PPGED) e Programa de Pós-graduação em Segurança Pública (PPGSP). É coordenadora do Núcleo de Estudos Aplicados a Comportamento, do PPGED da UFPA. E-mail: maelyramos@hotmail.com

\section{iD http://orcid.org/0000-0001-6150-6345}

\section{Edson Marcos Leal Soares Ramos}

Doutor em Engenharia de Produção pela Universidade Federal de Santa Catarina (UFSC). Professor Titular do Programa de Pós-graduação em Segurança Pública (PPGSP), da Universidade Federal do Pará. Membro do Núcleo de Estudos Aplicados ao Comportamento (NEAC). E-mail: ramosedson@gmail.com

(iD http://orcid.org/0000-0001-5425-8531 